\title{
Desempenho, características de carcaça, qualidade da carne e condição sanitária de suínos criados nas fases de crescimento e terminação nos sistemas confinado convencional e de cama sobreposta
}

\author{
Performance, carcass characteristics, meat quality and health condition of growing and finishing \\ pigs raised in conventional and deep litter housing systems
}

\author{
Osmar Antonio Dalla Costa ${ }^{I^{*}}$ Armando Lopes do Amaral ${ }^{\mathrm{I}}$ Jorge Vitor Ludke $^{\mathrm{I}}$ \\ Arlei Coldebella' Elsio Antonio Pereira de Figueiredo ${ }^{I}$
}

RESUMO

O objetivo deste trabalho foi avaliar o efeito do sistema de alojamento sobre o desempenho zootécnico, as características da carcaça, a qualidade da carne e a condição sanitária de suínos criados em seis diferentes sistemas. Foram usados 803 suínos mestiços MS58 x (Landrace x Large White) em sete lotes seqüenciais no tempo, avaliando as fases de creche e de crescimento-terminação. $\mathrm{Na}$ creche, foram avaliados três tipos de alojamento: confinado convencional (CO); cama sobreposta-(CS); e sistema intensivo de suínos criados ao ar livre (SISCAL). No crescimento-terminação, foram avaliados apenas dois tipos de alojamento (CO e CS), sendo o último subdividido em três substratos (palha de trigoPT, casca de arroz-CA e serragem-SE). Para a análise estatística foi considerado no modelo os efeitos de lote, o tratamento (combinação de tipos de alojamento na creche e no crescimento-terminação), idade dos suínos (84, 105, 126, 147 e 160 dias) e a interação tratamento $x$ idade. Suínos alojados no sistema CO apresentaram maior $(P<0,05)$ ganho de peso diário (824 vs. $779 g$ ), maior peso de carcaça quente (75 vs. $69 \mathrm{~kg}$ ) e maior espessura de toucinho (18, vs. $17 \mathrm{~mm})$ do que os criados sobre cama todavia estes suínos apresentaram peso do conteúdo estomacal significativamente $(P<0,05)$ maior $(360$ vs. 204g) e menor grau de lesão do estômago do que aqueles alojados em tratamento convencional, embora com maior prevalência de espirro. O sistema de alojamento não afetou os aspectos sanitários avaliados, contudo, suínos alojados em cama sobreposta no crescimento-terminação apresentaram desempenho e características de carcaça inferiores àqueles do sistema convencional, independentemente do sistema de alojamento utilizado na creche.

Palavras-chave: cama sobreposta, características de carcaça, desempenho, qualidade da carne, sanidade, SISCAL, alojamento.

\section{ABSTRACT}

The objective of this study was to evaluate the effect of housing system on live performance, carcass characteristics, meat quality, and the sanitary condition of pigs raised in six different systems. A total of 803 MS58 x (Landrace $x$ Large White) hybrid type pigs was used in seven subsequent lots, during the nursery and growing-finishing phases. At the nursery period, the systems evaluated were: conventional (CO), deep litter (CS), and intensive free range (SISCAL), while during the growing-finishing phase only the CO and CS systems were evaluated, being the CS system subdivided into three substrates (wheat straw, rice hulls and wood shaving). The factors considered on the statistical analysis were lot, treatment (combination of housing type used during nursery and growingfinishing phases), age (84, 105, 126, 147 e 160 days), and the interaction treatment $x$ age. A significant effect of housing type was observed on weight gain, carcass characteristics, and weight of heart, liver, full stomach and stomach content. Animals housed on conventional treatment had higher $(P<0.05)$ weight gain (824 vs. $779 \mathrm{~g}$ ), higher hot carcass weight (75 vs. $69 \mathrm{~kg}$ ) and higher fat thickness (18 vs. $17 \mathrm{~mm}$ ) than the ones raised on deep litter, however, these, on the other hand, presented larger $(P<0.05)$ gut content (360 vs. 204g) and less degree of gut lesion than those of the conventional treatment, eventhough with higher sneeze prevalence. The housing system did not influence the health, however pigs housed on deep litter system during the growing-finishing phase presented lower performance and carcass values than the ones housed in the conventional housing system despite the housing system used during the nursery phase.

Key words: carcass traits, deep litter, health condition, intensive free range, nursery, performance, meat quality, housing.

IEmbrapa Suínos e Aves, CP 21, 89700-000, Concórdia, SC, Brasil. E-mail: osmar@cnpsa.embrapa.br. *Autor para correspondência. 


\section{INTRODUÇÃO}

A suinocultura da região Sul do Brasil é uma atividade desenvolvida geralmente em pequenas propriedades rurais que, na sua grande maioria, ainda emprega mão-de-obra tipicamente familiar, constituindo uma importante fonte de renda e de estabilidade social. Contudo, a atividade tem sido caracterizada como sendo de grande impacto ambiental. Os efeitos sobre o meio ambiente decorrem, principalmente, do sistema de alojamento utilizado para os suínos, favorecendo a produção de dejetos líquidos nos alojamentos em pisos compacto ou semi-ripado largamente utilizados. Nessas condições, exige-se a utilização de esterqueiras ou lagoas para armazenamento dos dejetos, o que tem dificultado a manutenção dos produtores na atividade e o incremento da produção de suínos em algumas regiões (OLIVEIRA et al., 1998; OLIVEIRA, 2004).

Com objetivo de diminuir os impactos ambientais da suinocultura e maximizar a produção, novas tecnologias para o alojamento dos animais são propostas de forma continuada. Entre elas podemos citar o alojamento em cama sobreposta (CS), que consiste na utilização de um leito profundo composto por um substrato (maravalha, palha de cereais, casca de arroz, entre outros) com a função de absorver a fração líquida dos dejetos produzidos pelos animais, durante o período de permanência desses na unidade. Este tipo de alojamento é uma alternativa para a produção de suínos devido ao baixo custo de implantação das instalações, à facilidade de manejo, ao conforto e ao bem-estar animal em algumas épocas do ano. Também apresenta vantagens quanto ao manejo dos dejetos em regiões declivosas, do oeste catarinense, naquelas com produção agrícola intensiva (fruticultura e olericultura, principalmente) que demanda adubo orgânico de boa qualidade.

Alguns estudos foram realizados para avaliar o impacto dos sistemas de produção dos suínos no desempenho zootécnico, sanitário, qualidade da carne e do bem-estar animal. PERDOMO et al. (1997) observaram que o desempenho dos suínos alojados em cama sobreposta de palha foi inferior aos alojados no tratamento convencional (CO), sugerindo que o desempenho inferior deve-se ao desconforto gerado pelo acúmulo de lodo na baia, em função da sua baixa capacidade de absorção da água. OLIVEIRA (1999), em estudo comparativo entre dois sistemas de alojamento, convencional (piso ripado) e cama sobreposta (maravalha), afirmou não haver diferença no desempenho zootécnico dos suínos nem no estado sanitário dos animais alojados em ambiente termoneutro. CORRÊA et al. (2000) não encontraram diferença no ganho de peso, no consumo de ração e água, no estado sanitário e nas características de carcaça entre animais alojados em CO x CS (maravalha, serragem, sabugo de milho e casca de arroz). Já BRIDI et al. (2003a) verificaram que os suínos alojados no sistema ao ar livre apresentaram desempenho e características de carcaça inferiores quando comparados aos alojados no CO e CS. Entretanto, BRIDI et al. (2003b) não encontraram diferença entre os sistemas de produção (convencional, cama e ao ar livre) na qualidade de carcaça.

O objetivo deste trabalho foi avaliar o efeito do sistema de produção de suínos sobre o desempenho zootécnico, as característica da carcaça, a qualidade da carne, o peso das vísceras e a situação sanitária dos animais.

\section{MATERIAL E MÉTODOS}

O experimento foi conduzido na Embrapa Suínos e Aves, em Concórdia, SC, de dezembro de 2000 a fevereiro de 2002. Foram utilizados 803 leitões mestiços MS58 x (LD x LW), alojados na fase de creche em três tipos de alojamento: sistema intensivo de suínos criados ao ar livre (SISCAL); confinados em cama sobreposta; e confinados em piso de concreto ripado (convencional), em sete lotes no tempo, cujos tratamentos foram compostos por seis combinações entre três tipos de alojamento em duas fases de criação (creche e crescimento-terminação), conforme a tabela 1. As rações utilizadas foram formuladas para atender as exigências nutricionais nas respectivas fases de criação (três idades na creche e cinco idades no crescimento-terminação). Na fase de creche, a unidade experimental foi composta por 40 leitões sistema $^{-1}$ lote $^{-1}$, sendo que na fase de crescimentoterminação esses leitões foram divididos em dois grupos: 10 leitões foram alojados no sistema convencional e os outros 30 leitões foram alojados no sistema de cama sobreposta (serragem, casca de arroz e palha de trigo).

Foram utilizadas seis instalações de cama sobreposta (duas de cada substrato), modelo Embrapa (DALLA COSTA et al., 2006), e dois galpões convencionais com piso semi-ripado da Unidade de crescimento e terminação do "Sistema de Produção de Suínos" da Embrapa Suínos e Aves. Na fase de crescimento-terminação para os alojamento em cama sobreposta, utilizou-se área de $1,3 \mathrm{~m}^{2}$ suíno $^{-1}$ subdividido em $1,05 \mathrm{~m}^{2}$ suíno $^{-1}$ de substrato (área livre) e $0,25 \mathrm{~m}^{2}$ suíno-1 $\mathrm{com}$ área de alimentação de piso de madeira. Já no alojamento CO os animais foram mantidos em baias coletivas, com piso semi-ripado, com 
Tabela 1 - Esquema básico do delineamento experimental para os sistemas de alojamento de suínos na creche (sistema intensivo de suínos ao ar livre - SISCAL, sistema confinado convencional e sistema suíno sobre cama sobreposta - SCP) e crescimento-terminação.

\begin{tabular}{llll}
\hline Creche & Crescimento e Terminação & Tratamento & Sigla \\
\hline \multirow{2}{*}{ Convencional } & Convencional & CO - CO & CO - CO \\
& SCP (Serragem)** & CO - Serragem & CO- SE \\
Ao ar livre (SISCAL) & Convencional & SISCAL - CO & SISCAL-CO \\
SCP (Palha de trigo)* & SCP (Casca de Arroz)** & SISCAL - Arroz & SISCAL-AR \\
& Convencional & Palha - CO CO & PA - PA \\
\hline
\end{tabular}

* Sistema de produção proposto por DALLA COSTA et al. (2001);

** Sistema de produção proposto por DALLA COSTA et al. (2006).

capacidade para alojar cinco suínos, e utilizou-se área de $1,3 \mathrm{~m}^{2}$ suíno $^{-1}$.

No sistema com palha de trigo, inicialmente foi colocada uma camada de $30 \mathrm{~cm}$ de altura e à medida que a cama ficava saturada era adicionada palha novamente. Quando havia a retirada dos suínos para o abate, esta palha era toda revolvida do galpão. Nos sistemas de casca de arroz e serragem foi colocada cama de $50 \mathrm{~cm}$ de altura.

A avaliação do desempenho dos animais (ganho de peso, consumo de ração e conversão alimentar) foi realizada a cada 14 dias. As características das carcaças foram avaliadas com o uso do aparelho HENNESSY GRADE PROBE (HGP-4), com o qual foram medidas a espessura de toucinho (ESPTO), a profundidade do músculo (PMU) e foi estimada a porcentagem de carne magra na carcaça (PCM). O peso da carcaça quente (PCQ) foi medido e o valor foi armazenado eletronicamente, utilizando balança eletrônica com capacidade de $300 \mathrm{~kg}$ e divisão de $100 \mathrm{~g}$. Para avaliar a qualidade da carne, foi medido o $\mathrm{pH}$ na meia carcaça esquerda nos músculos Longissimus dorsi (LD) e Semimembranosus (SM) 45 minutos $\left(\mathrm{pH}_{1}\right)$ e 24 horas após o abate $\left(\mathrm{pH}_{\mathrm{U}}\right)$. Para tanto, foi utilizado o pHmetro portátil. A avaliação da cor do músculo LD (C_LD) foi realizada com o auxílio de um padrão fotográfico ( 1 = rosa muito pálido; 2 = rosa pálido; 3 = rosada cinzento; $4=$ vermelho escuro e 5 = vermelho muito escuro).

Para avaliação clínica de pneumonia e rinite atrófica, foi realizada a contagem de tosse e espirro a cada 14 dias em 100\% dos animais, para estimar o índice de pneumonia (IP) e o índice de rinite atrófica (IRA) (MORÉS et al., 2001). A avaliação anatomopatológica de pulmões e cornetos nasais ao abate foi realizada em 100\% dos animais e também foi usada para estimar o IP e o IRA(LOPEZ et al., 1998). A avaliação dos cornetos nasais, dos pulmões e das lesões gástricas foi realizada de acordo com MORÉS et al. (2000). Foi feita também avaliação quanto ao escore de úlcera gástrica (LOPEZ et al., 1998) e à presença de lesões macroscópicas de linfadenite nos linfonodos submandibulares e mesentéricos. O peso do coração, dos rins, do fígado, dos pulmões e do estômago cheio e vazio foi avaliado nos três primeiros lotes.

A análise estatística do ganho de peso médio diário (GPMD) e da conversão alimentar (CA) acumulados, a partir dos 63 dias de idade, foi realizada utilizando-se a teoria de modelos mistos para medidas repetidas, considerando-se os efeitos de lote, o tratamento (combinação de tipo de alojamento com fase da criação), a idade dos suínos (84, 105, 126, 147 e 160 dias), a interação tratamento $\mathrm{x}$ idade e os 16 tipos de estruturas de matriz de variâncias e covariâncias, usando o PROC MIXED do SAS (2003). As comparações de interesse foram efetuadas por meio de cinco contrastes ortogonais, conforme o esquema do delineamento experimental no Quadrado 1.

Para demanda d’água na fase de crescimento-terminação, também foi usada a mesma lógica dos contrastes. Nesta fase foram comparados apenas os tratamento que envolviam a cama sobreposta, uma vez que não foi possível realizar as medições nos demais tratamentos. Dessa maneira, os contrastes utilizados para efetuar as comparações de interesse foram apenas os contrastes C1, C4 e C5.

A análise estatística da ocorrência de tosse/ espirro foi realizada por meio da análise da variância, considerando-se os efeitos de lote e os sistemas de alojamento durante o crescimento-terminação (pelo mesmo motivo da demanda d’água), sendo o desdobramento da análise igual àquele da demanda d’água. Para as demais variáveis, foi realizada a análise da variância, considerando os efeitos de lote e tratamento (combinação de sistemas de criação na creche e no crescimento-terminação), detalhando-se a análise por meio dos contrastes ortogonais descritos para o caso do GPMD e CA. A análise da mortalidade e do grau de lesão do estômago foi realizada por meio da 
regressão logística, considerando os efeitos de lote e tratamento.

\section{RESULTADOS E DISCUSSÃO}

Houve efeito $(\mathrm{P}<0,05)$ de tratamentos e de idade no ganho de peso médio diário dos suínos (GPMD) e somente de idade na conversão alimentar. Os suínos alojados em sistema convencional durante o crescimentoterminação apresentaram melhor (827g) GPMD $(\mathrm{P}<0,05)$ do que os suínos alojados em cama sobreposta, serragem $787 \mathrm{~g}$, casca de arroz $760 \mathrm{~g}$ e palha de trigo 696g, independente do tipo de alojamento na creche.

Entre aqueles suínos com crescimentoterminação convencional, não houve diferenças significativas $(\mathrm{P}>0,05)$ entre os tipos de alojamento na creche, enquanto que, para aqueles alojados em cama sobreposta, os suínos que vieram de creche convencional e de cama sobreposta de serragem apresentaram GPMD superior $(\mathrm{P}<0,05)$ em comparação com aqueles que vieram de creche no SISCAL e na palha de trigo. Destes dois últimos, a creche em palha de trigo apresentou os piores resultados (Figura 1). Os resultados deste estudo estão de acordo com os obtidos por PERDOMO et al. (1997), que também observaram que os suínos alojados em cama sobreposta de palha apresentaram desempenho inferior aos alojados em cama de serragem ou no sistema convencional. CORDEIRO, (2003), avaliando os sistemas de alojamento em cama sobreposta (maravalha e casca de arroz), não encontrou efeito do sistema de alojamento na fase de crescimento (25-75kg) para ganho de peso. Todavia, observou que os suínos alojados sobre a casca de arroz apresentaram desempenho $(\mathrm{P}<0,05)$ inferior aos alojados no sistema convencional durante a fase de terminação (76-120kg).
Contudo, os resultados aqui apresentados diferem dos encontrados por OLIVEIRA (1999), CORRÊA et al. (2000), BRIDI et al. (20003a) e MOREIRA et al. (2003), que não encontraram efeito dos sistemas de alojamento de suínos no ganho de peso dos animais. Inúmeros fatores condicionam o melhor desempenho dos suínos no sistema convencional, dos quais podem ser relacionados os aspectos sanitários, o conforto térmico (o alojamento de suínos sobre cama geralmente favorece no inverno, pois a produção de calor deste sistema é maior devido à fermentação da cama e de dejetos e à ingestão de sobre (cama) pelos suínos.

Não foi observado efeito $(\mathrm{P}>0,05)$ dos tratamentos no $\mathrm{pH}_{1}, \mathrm{pH}_{\mathrm{U}}$ dos músculos LD e SM (Tabela 2). Os resultados obtidos no presente estudo estão de acordo com os encontrados por BRIDI et al. (2003b), que não encontraram efeito do sistema de alojamento de suínos (convencional, cama sobreposta e SISCAL) sobre os parâmetros de qualidade da carne aqui avaliados.

Verificou-se efeito $(\mathrm{P}<0,05)$ dos tratamentos sobre ESPTO, PMU, PCM e PCQ, sendo que suínos em crescimento-terminação alojados no sistema convencional apresentaram maiores valores de ESPTO, PMU e PCQ do que suínos alojados em cama sobreposta, independentemente do tipo de creche. Conseqüentemente, suínos em crescimento-terminação alojados no sistema convencional apresentaram menor porcentagem de carne magra na carcaça do que os suínos alojados sobre cama.

Esses resultados diferem dos obtidos por OLIVEIRA (1999) e CORRÊA et al. (2000), que não observaram efeito dos sistemas de alojamento (serragem, sabugo de milho e casca de arroz) sobre as características de carcaça dos suínos. Além disso, LOPES (2004), avaliando os sistemas convencional e

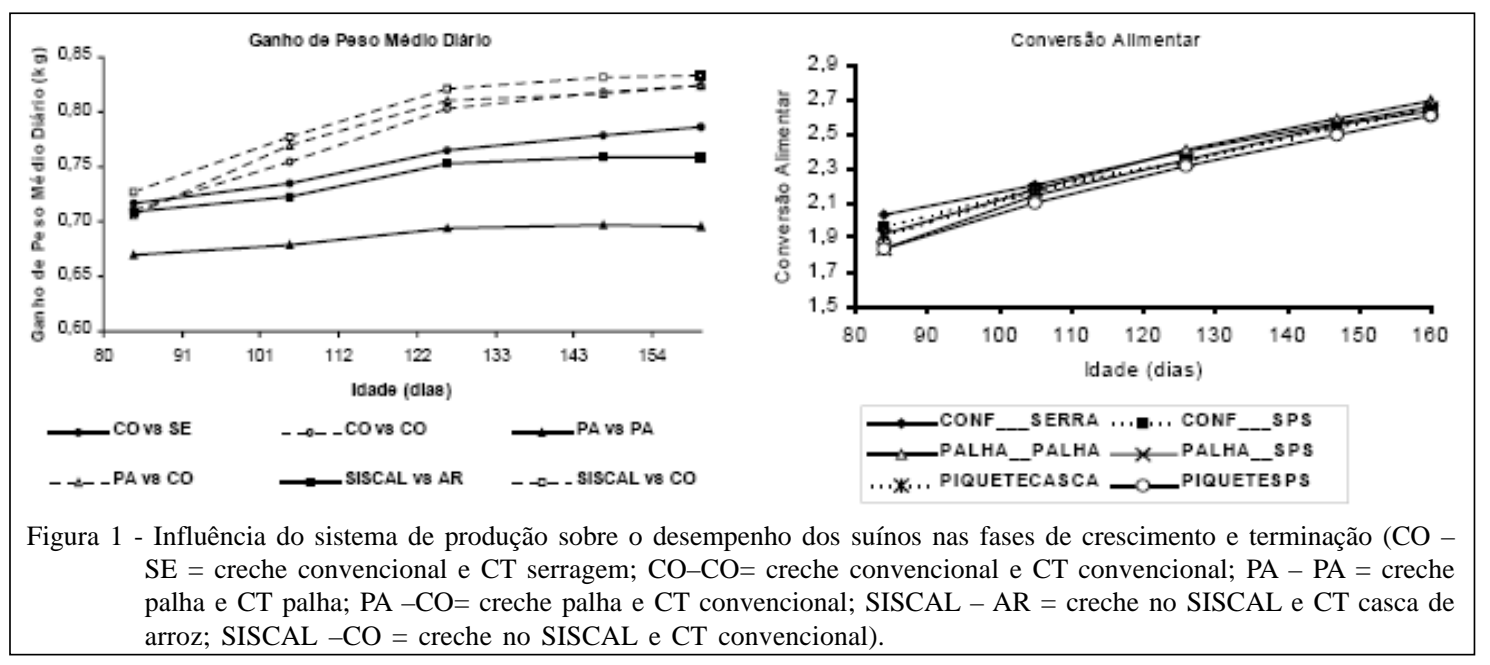

Ciência Rural, v.38, n.8, nov, 2008. 
Tabela 2 - Características da carcaça e da qualidade da carne e peso das vísceras dos suínos submetidos aos sistemas de alojamento na fases de crescimento-terminação.

\begin{tabular}{|c|c|c|c|c|c|c|c|}
\hline \multirow{2}{*}{ Variáveis* } & \multirow[b]{2}{*}{$\mathrm{CO}-\mathrm{SE}^{*}$} & \multirow[b]{2}{*}{$\mathrm{CO}-\mathrm{CO}^{*}$} & \multirow[b]{2}{*}{$\mathrm{PA}-\mathrm{PA}^{*}$} & \multirow[b]{2}{*}{$\mathrm{PA}-\mathrm{CO}^{*}$} & \multirow[b]{2}{*}{ SISCAL - AR* } & \multirow[b]{2}{*}{ SISCAL - CO* } & \multirow[b]{2}{*}{ Teste Anova } \\
\hline & & & & & & & \\
\hline ESPTO & $15,51 \pm 0,47$ & $17,71 \pm 0,47$ & $14,55 \pm 0,47$ & $17,85 \pm 0,47$ & $14,61 \pm 0,47$ & $17,72 \pm 0,47$ & 0,0001 \\
\hline PMU & $57,34 \pm 0,95$ & $58,13 \pm 0,95$ & $52,40 \pm 0,95$ & $56,97 \pm 0,95$ & $57,90 \pm 0,95$ & $57,46 \pm 0,95$ & 0,0016 \\
\hline PCM & $57,10 \pm 0,40$ & $56,02 \pm 0,40$ & $56,65 \pm 0,40$ & $55,71 \pm 0,40$ & $57,74 \pm 0,40$ & $55,88 \pm 0,40$ & 0,0082 \\
\hline PCQ & $69,63 \pm 0,89$ & $74,59 \pm 0,89$ & $62,00 \pm 0,89$ & $74,19 \pm 0,89$ & $66,89 \pm 0,89$ & $74,42 \pm 0,89$ & 0,0001 \\
\hline $\mathrm{pH}_{1} \mathrm{SM}$ & $6,15 \pm 0,05$ & $6,13 \pm 0,05$ & $6,21 \pm 0,05$ & $6,24 \pm 0,05$ & $6,24 \pm 0,05$ & $6,24 \pm 0,05$ & 0,4786 \\
\hline $\mathrm{PH}_{1} \mathrm{LD}$ & $6,02 \pm 0,05$ & $6,00 \pm 0,05$ & $6,07 \pm 0,05$ & $6,08 \pm 0,05$ & $6,09 \pm 0,05$ & $6,07 \pm 0,05$ & 0,8136 \\
\hline $\mathrm{pH}_{\mathrm{U}} \mathrm{SM}$ & $5,72 \pm 0,03$ & $5,62 \pm 0,03$ & $5,66 \pm 0,03$ & $5,62 \pm 0,03$ & $5,69 \pm 0,03$ & $5,64 \pm 0,03$ & 0,1829 \\
\hline $\mathrm{pH}_{\mathrm{U}} \mathrm{LD}$ & $5,61 \pm 0,03$ & $5,53 \pm 0,03$ & $5,55 \pm 0,03$ & $5,53 \pm 0,03$ & $5,60 \pm 0,03$ & $5,53 \pm 0,03$ & 0,0671 \\
\hline C_LD & $2,61 \pm 0,07$ & $2,52 \pm 0,07$ & $2,52 \pm 0,07$ & $2,52 \pm 0,07$ & $2,58 \pm 0,07$ & $2,55 \pm 0,07$ & 0,9124 \\
\hline PCOR & $332,5 \pm 4,74$ & $354,4 \pm 4,74$ & $289,4 \pm 4,74$ & $345,4 \pm 4,74$ & $309,8 \pm 4,74$ & $349,6 \pm 4,74$ & 0,0001 \\
\hline PRIM & $303,4 \pm 10,4$ & $316,2 \pm 10,4$ & $267,6 \pm 10,4$ & $306,2 \pm 10,4$ & $279,1 \pm 10,4$ & $303,5 \pm 10,4$ & 0,0573 \\
\hline PPUL & $791,3 \pm 26,9$ & $828,9 \pm 26,9$ & $714,8 \pm 26,9$ & $778,4 \pm 26,9$ & $772,7 \pm 26,9$ & $793,3 \pm 26,9$ & 0,1742 \\
\hline PFIG & $1388 \pm 31,4$ & $1518 \pm 31,4$ & $1245 \pm 31,4$ & $1451 \pm 31,4$ & $1277 \pm 31,4$ & $1510 \pm 31,4$ & 0,0003 \\
\hline PESCH & $1017 \pm 72,2$ & $718,0 \pm 72,2$ & $931,4 \pm 72,2$ & $679,1 \pm 72,2$ & $977,4 \pm 72,2$ & $749,5 \pm 72,2$ & 0,0271 \\
\hline PESVA & $534,4 \pm 11,7$ & $514,1 \pm 11,7$ & $490,6 \pm 11,7$ & $499,3 \pm 11,7$ & $509,2 \pm 11,7$ & $518,8 \pm 11,7$ & 0,2171 \\
\hline PCES & $482,9 \pm 65,6$ & $203,9 \pm 65,6$ & $440,8 \pm 65,6$ & $179,8 \pm 65,6$ & $470,5 \pm 65,6$ & $230,7 \pm 65,6$ & 0,0168 \\
\hline
\end{tabular}

$\mathrm{CO}$ - SE = creche convencional e CT serragem; $\mathrm{CO}$ - CO = creche convencional e CT convencional; $\mathrm{PA}-\mathrm{PA}=$ creche palha e CT palha; $\mathrm{PA}$ - CO = creche palha e CT convencional; SISCAL - AR = creche no SISCAL e CT casca de arroz; SISCAL - CO = creche no SISCAL e CT convencional. ESPTO = Espessura de toucinho (mm); PMU= Profundidade do Longissimus dorsi (mm); PCM = Porcentagem de carne; $\mathrm{PCQ}=$ Peso da carcaça $(\mathrm{kg}) ;\left(\mathrm{pH}_{1} \mathrm{SM}\right)$ e $\left(\mathrm{pH}_{1} \mathrm{LD}\right)=\mathrm{pH} 45$ minutos após o abate dos músculos dos Semimembranosus (SM) e Longissimus dorsi; $\left(\mathrm{pH}_{\mathrm{u}} \mathrm{SM}\right)$ e $\left(\mathrm{pH}_{1} \mathrm{LD}\right)=\mathrm{pH} 24$ horas após o abate dos músculos dos Semimembranosus (SM) e Longissimus dorsi; C_LD = cor do músculo Longissimus dorsi, PCOR= Peso do coração; PRIM= Peso do rim; PPUL= Peso do pulmão; PFIG = Peso do fígado; PESCH = Peso do estômago cheio; PESVA = Peso do estômago vazio; PCES = Peso do conteúdo estômago.

* Médias e erros padrão

cama sobreposta com serragem e casca de arroz, não verificou efeito do sistema de alojamento sobre as características da carcaça.

Suínos alojados no sistema de creche convencional quando passaram para a fase de crescimento-terminação em cama sobreposta de serragem apresentaram maiores valores de PCQ do que os alojados em palha de trigo ou no SISCAL. Os suínos alojados em creche no SISCAL, quando passaram para o crescimento-terminação na cama com casca de arroz, apresentaram maiores valores de PMU e PCQ do que os alojados em palha de trigo durante ambas as fases de creche e crescimento-terminação. Estas diferenças PCQ, PMU e ESPTO estão relacionadas com o peso dos suínos no final do estudo.

Em relação à demanda de diária de água (DDA), não foi observado efeito $(\mathrm{P}>0,05)$ do sistema de alojamento no crescimento-terminação nem da interação sistema de alojamento no crescimentoterminação $\times$ idade. Este resultado está de acordo com os obtidos por CORRÊA et al. (2000), que também não encontraram efeito do sistema de alojamento sobre o consumo de água dos suínos. Os valores médios de DDA (13,25 litros/animal/dia) do presente estudo ficaram acima dos obtidos por OLIVEIRA (2003). CORDEIRO (2003) verificou na fase terminação-II (105$120 \mathrm{~kg}$ ) que os suínos alojados sobre cama de casca de arroz consumiram menos água do que os alojados sobre maravalha. OLIVEIRA (2003), comparando o consumo diário de água entre os sistemas de alojamento de suínos com cama de maravalha ou piso ripado em ambiente com temperatura controlada $\left(22^{\circ} \mathrm{C}\right)$, conclui que o consumo médio foi de 5,22 e 4,96L suíno-1 dia, respectivamente, para cama de maravalha e piso ripado, valores estes muito inferiores aos encontrados no presente estudo (13,25 $\left.\mathrm{L}_{\text {animal }}{ }^{-1} \mathrm{dia}^{-1}\right)$. Adiferença entre os dois estudos provavelmente está associada ao efeito da temperatura ambiente do sistema de cama onde os suínos alojados neste sistema podem ter sido submetidos a maior estresse térmico provocado pela fermentação da cama.

A média de mortalidade foi de 1,77\%, com intervalo de confiança de $95 \%$ de $1,05 \%$ a 3,01\%, sendo que não foi observado efeito $(\mathrm{P}>0,05)$ dos tratamentos sobre ela.

O sistema de alojamento adotado no crescimento-terminação não influenciou $(\mathrm{P}>0,05)$ a porcentagem de ocorrência de tosse. Contudo, 
influenciou $(\mathrm{P}<0,05)$ a porcentagem de ocorrência de espirro. Suínos alojados no sistema convencional durante o crescimento-terminação apresentaram menor prevalência de espirro (1,35\% versus 5,42\%) do que os alojados sobre cama e esses últimos não diferiram $(\mathrm{P}>0,05)$ entre si. Os resultados diferem dos obtidos por CORRÊA et al. (2000), que encontraram efeito do sistema de alojamento sobre a porcentagem de tosse, com animais no sistema convencional apresentando maiores valores do que os suínos da cama sobreposta de resíduo de milho.

Foi verificado efeito $(\mathrm{P}<0,05)$ dos tratamentos sobre o grau de lesão do estômago, sendo que os animais alojados no sistema convencional durante o crescimento-terminação apresentaram maior prevalência de paraqueratose e de úlcera gástrica no estômago do que os alojados sobre cama, independente do sistema de creche. Esses resultados estão de acordo com OLIVEIRA (1999), que encontrou efeito significativo $(P<0,05)$ sobre o grau de lesão do estômago, concluindo que $70 \%$ dos animais alojados sobre cama de maravalha não apresentaram qualquer tipo de lesão e os $30 \%$ restantes apresentaram somente paraqueratose. Entretanto, no sistema de piso ripado, somente 30\% dos animais não apresentaram lesão do estômago, sendo que os demais (70\%) apresentaram paraqueratose e úlcera gástrica no estômago. Já CORRÊA et al. (2000) não encontraram efeito dos sistemas de alojamento dos suínos sobre o grau de lesão do estômago. Os tratamentos não influenciaram $(\mathrm{P}>0,05)$ os índices de pneumonia nem de rinite atrófica progressiva. Esses resultados validam os obtidos por OLIVEIRA (1999) e CORRÊA et al. (2000).

Não foi observada durante o crescimentoterminação ocorrência de linfadenite nos suínos alojados no sistema convencional nem nos sistema de cama sobreposta de casca de arroz e palha de trigo. Contudo, nos suínos alojados em cama sobreposta de serragem, houve prevalência de 1,9\% de linfadenite. Esse último valor é inferior aos obtidos por CORRÊA et al. (2000) e SILVA et al. (2001), porém, está acima do encontrado por MORES (1997) em frigorífico da região.

Não se observou efeito $(\mathrm{P}>0,05)$ do sistema de alojamento sobre o peso dos rins, dos pulmões e do estômago vazio. Contudo observou-se efeito $(\mathrm{P}<0,05)$ nos pesos do coração, do fígado, do estômago cheio e do conteúdo estomacal (Tabela 2). Durante o crescimento-terminação, suínos alojados no sistema convencional apresentaram maiores pesos de coração, fígado e carcaça quente do que os alojados em cama sobreposta, enquanto estes últimos apresentaram maiores valores de estômago cheio e conteúdo estomacal do que os primeiros. Isso pode estar relacionado com jejum pré-abate, pois animais mantidos no sistema de cama podem consumir este tipo de material, o que não é possível no alojamento convencional.

Suínos alojados em cama sobreposta de serragem apresentaram maiores valores do peso do coração, do fígado, do estômago cheio e do conteúdo estomacal do que os alojados sobre palha de trigo e casca de arroz, sendo que os alojados em cama sobreposta de casca de arroz apresentaram maiores valores do peso do coração, do fígado, do estômago cheio e do conteúdo estomacal do que os alojados sobre palha de trigo.

A viabilização técnica e a econômica do alojamento de suínos em cama sobreposta, nas condições climáticas do Sul do Brasil, estão relacionadas principalmente aos aspectos sanitários (oriundos da presença de lesões macroscópicas de linfadenite), do manejo da cama e da redução do estresse térmico oriundo da fermentação do substrato da cama.

\section{CONCLUSÕES}

Os sistemas de alojamento não afetaram os aspectos sanitários avaliados. No entanto, suínos alojados em cama sobreposta no crescimentoterminação apresentaram desempenho inferior aqueles do sistema convencional, independentemente do sistema de alojamento utilizado na creche.

\section{REFERÊNCIAS}

BRIDI, A.M. et al. Efeito do genótipo halotano e de diferentes sistemas de produção no desempenho produtivo e na qualidade da carcaça suína. Revista da Sociedade Brasileira de Zootecnia, v.32 n.4, p.942-950, 2003a.

BRIDI, A.M. et al. Efeito do genótipo halotano e de diferentes sistemas de produção na qualidade da carne suína. Revista da Sociedade Brasileira de Zootecnia, v.32 n.6, p.1362-1370, 2003b.

CORDEIRO, M.B. Avaliação de sistema de cama sobreposta quanto ao conforto térmico e ambiental e ao desempenho zootécnico para suínos das fases de crescimento e terminação. 2003. 63f. Dissertação (Mestrado em Engenharia Agrícola) - Universidade Federal de Viçosa, Viçosa.

CORRÊA, É.K. et al. Condicionamento ambiental e desempenho de suínos em crescimento e terminação criados sobre piso com leito de cama. Revista Sociedade Brasileira de Zootecnia, v.29, n.6, p.2072-79, 2000 (Suplemento 1).

DALLA COSTA O.A. et al. Sistema intensivo de suínos criados ao ar livre: creche móvel sobre cama para suínos Concórdia: Embrapa Suínos e Aves, 2001. 68p. (Embrapa Suínos e Aves. Comunicado Técnico 278). 
DALLA COSTA O.A. et al. Sistema criação sobre cama modelo embrapa. Concórdia: Embrapa Suínos e Aves, 2006. 6p. (Embrapa Suínos e Aves. Comunicado Técnico 419).

LOPEZ, A.C. et al. ProAPA-suínos: Programa para avaliação patológica no abate de suínos - guia do usuário. Concórdia: EMBRAPA-CNPSA, 1998. 64p. (EMBRAPACNPSA, Documentos, 49).

LOPES, E.J.C. Analise do bem-estar e desempenho de suínos em sistema de cama sobreposta. 2004. 99f. Dissertação (Mestrado Agroecossistema) - Universidade Federal de Santa Catarina.

MOREIRA, I. et al. Desempenho e características de carcaça de suínos (33 - 84kg) criados em baias de piso compacto ou com lâmina d'água. Revista da Sociedade Brasileira de Zootecnia, v.32 n.1, p.132-139, 2003.

MORÉS, N. Linfadenite em suínos causados por microbactérias atípicas. In: CONGRESSO BRASILEIRO DE VETERINÁRIOS ESPECIALISTA EM SUÍNOS, 8., 1997, Foz do Iguaçu. Pr. Anais... Concórdia: Embrapa - CNPSA, 1997. p.165-172.

MORÉS, N. et al. Avaliação patológica de suínos no abate manual de identificação. Brasília: EMBRAPA Comunicação para Transferência de tecnologia, 2000. 40p.

MORÉS, N. et al. Estimativa dos índices de pneumonia, pela tosse, e de rinite atrófica, por espirros, em suínos. Arquivo Brasileiro Medicina Veterinária e Zootecnia, v.53, n.3, p.284-289, 2001a.

OLIVEIRA, P.A.V. de. et al. Comparasion de l'évaporation d'éau en élevage de porcs procs surs litière de sciure ou caillebotis intégral. Journées Rech. Porcine en France, v.30, p.355-361, 1998. OLIVEIRA, P.A.V de. Comparasion des systèmes d'élevage des procs surs litière de sciure ou caillebotis intégral. 1999. 263f. (These de Docteur) - RENNES:ENSA.

OLIVEIRA, P.A.V. de. Modelo matemático para estimar a evaporação d'água contida nos dejetos, em sistemas de criação de suínos sobre cama de maravalha e piso ripado, nas fases de crescimento e terminação. Journal of the Brazilian Society of Agricultural Engineering, v.23, n.3, p.398626, 2003.

OLIVEIRA, P.A.V. de. Tecnologias para o manejo de resíduos na produção de suínos: manual de boas práticas. Concórdia: Embrapa Suínos e Aves, 2004. $109 \mathrm{p}$

PERDOMO, C.C. et al. Efeito do tipo de cama sobre o desempenho de suínos e, crescimento e terminação. In: CONGRESSO BRASILEIRO DE VETERINÁRIOS ESPECIALISTA EM SUÍNOS, 8., 1997, Foz do Iguaçu. Pr. Anais... Concórdia: Embrapa - CNPSA, 1997. p.421422.

SAS INSTITUTE INC. *System for Microsoft Windows:* release 9.1.3. Cary, NC, 2002-2003. 1 CD-ROM.

SILVA,V.S. et al. Dinâmica da infecção por Mycobacterium avium em suínos. In: CONGRESSO BRASILEIRO DE VETERINÁRIOS ESPECIALISTAS EM SUÍNOS, 10., 2001, Porto Alegre. Anais... Concórdia: Embrapa Suínos e Aves, 2001. V.2, p.137-138. 